Authors

David J. Jones, Scott A. Diddams, Matthew S. Taubman, Steven T. Cundiff, Long-Sheng Ma, and John L. Hall 


\title{
Frequency comb generation using femtosecond pulses and cross-phase modulation in optical fiber at arbitrary center frequencies
}

\author{
David J. Jones, Scott A. Diddams, Matthew S. Taubman, Steven T. Cundiff, Long-Sheng Ma, and John L. Hall \\ JILA, University of Colorado and National Institute of Standards and Technology, Boulder, Colorado 80309-0440
}

Received October 21, 1999

A technique is presented for generating optical frequency combs centered at arbitrary wavelengths by use of cross-phase modulation (XPM) between a femtosecond pulse train and a cw laser beam by copropagating these signals through an optical fiber. We report results from use of this method to place a $90-\mathrm{MHz}$ frequency comb on an iodine-stabilized Nd:YAG laser at $1064 \mathrm{~nm}$ and on a frequency-doubled $\mathrm{Nd}: \mathrm{YVO}_{4}$ laser at $532 \mathrm{~nm}$. XPM is verified to be the comb-generating process, and the width of the frequency comb is measured and compared with theory. The spacing of the frequency comb is compared with the femtosecond source, and a frequency measurement with this comb is demonstrated. (C) 2000 Optical Society of America

OCIS codes: $\quad 120.4800,120.3940,060.4370,060.7140,120.0120$.

A key tool for optical frequency metrology is a frequency comb that is capable of spanning frequency gaps greater than $100 \mathrm{GHz}$. In the past, frequency gaps of up to $\sim 5 \mathrm{THz}$ were bridged with optical frequency comb generators. ${ }^{1,2}$ Recently a second technique that uses the mode structure underneath the extremely broad spectral bandwidth of femtosecond (fs) pulses was used in a frequency chain to measure the cesium $D_{1}$ frequency $^{3}$ and to directly span the $104-\mathrm{THz}$ gap between existing optical standards at 778 and $1064 \mathrm{~nm}^{4}$ In this Letter we propose and demonstrate another application of fs technology to optical frequency metrology based on cross-phase modulation ${ }^{5}$ (XPM) in optical fiber, By simultaneously propagating the output of a cw laser and a fs pulse train through an optical fiber, a frequency comb with spacing equal to the frequency of the pulse train is written onto the cw laser by XPM. Because of the broadband nature of both silica and XPM, the range of possible center frequencies of a comb constructed in this manner is limited only by the availability of a cw laser and the guidance properties of the fiber.

Figure 1 details the experimental configuration. Approximately $120 \mathrm{~mW}$ of average power from a Kerr-lens mode-locked Ti:sapphire ${ }^{6}$ laser is coupled into a single-mode optical fiber. The output pulse is roughly $10 \mathrm{fs}$, and the spectral bandwidth is $115 \mathrm{~nm}$. The $90-\mathrm{MHz}$ repetition rate of the Ti:sapphire laser is stabilized to a $\mathrm{rf}$ synthesizer referenced to a rubidium oscillator with an instability of $2.5 \times 10^{-11}$ over an averaging time of $1 \mathrm{~s}$. Further details on the stabilization of the fs laser are reported elsewhere. ${ }^{4,7}$ It is important to note that it is necessary to stabilize only the pulse-repetition frequency and not the absolute optical frequency of the fs comb, because the center frequency of the comb generated by XPM is uniquely determined by the stabilized cw laser.

In the first set of experiments, $3 \mathrm{~mW}$ of a cw iodine$\left(\mathrm{I}_{2^{-}}\right)$stabilized Nd:YAG laser ${ }^{8}$ is launched into the same fiber as the fs laser. The fiber output is collimated and spectrally resolved by means of a grating, and an $\sim 1$-nm-wide spectral slice is focused onto a $\mathrm{P}-\mathrm{I}-\mathrm{N}$ diode. We confirm the absence of any $90-\mathrm{MHz}$ fs comb lines at $1064 \mathrm{~nm}$ by turning off the stabilized cw Nd:YAG laser and examining the rf spectrum. Subsequently, when the $\mathrm{I}_{2}$-stabilized cw Nd:YAG laser is turned on and launched into the fiber, a $90-\mathrm{MHz}$ comb is observed at $1064 \mathrm{~nm}$.

The new comb at $1064 \mathrm{~nm}$ is created by a thirdorder nonlinear process with the induced polarization $P \propto E_{i} E_{j} E_{k}{ }^{*}$, where $i, j$, and $k$ refer to either the cw laser or a fs mode. Two of the fields must be fs modes for a new comb to be generated; however, different results are obtained, depending on whether $E_{\mathrm{cw}}$ is the conjugated field. If it is not the conjugated field, the frequencies of the new comb are given by $f_{\text {comb }}=f_{\mathrm{cw}}+f_{m}-f_{n}=f_{\mathrm{cw}} \pm s \Omega_{r}$, where $f_{m, n}$ are two fs modes, $s$ is an integer, and $\Omega_{r}$ is the pulserepetition rate. This situation is known as XPM, and the new comb frequencies depend only on the spacing of the fs laser comb, not on its absolute frequency position. In the other case, when $E_{\mathrm{cw}}$ is conjugated, $f_{\text {comb }}=f_{m}+f_{n}-f_{\mathrm{cw}}=f_{\mathrm{cw}}+2 \delta \pm s \Omega_{r}$, where $\delta$ is the detuning between the cw laser and the fs comb. We denote this case four-wave mixing (FWM), although strictly speaking XPM is also one specific permutation of FWM. Note that the frequency of the comb generated by FWM depends on the absolute frequency of the fs comb.

To verify that the comb is created by XPM, we combine a second, tunable $\mathrm{cw} \mathrm{Nd}$ :YAG laser in free space with the spectrally filtered output from the

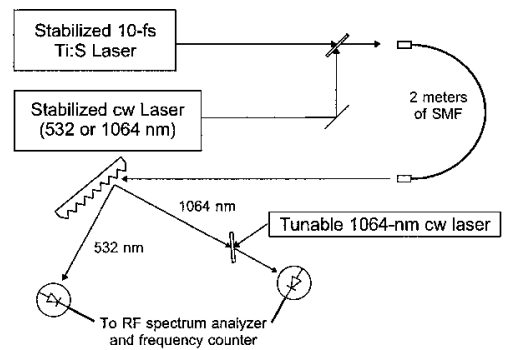

Fig. 1. Experimental setup: SMF, single-mode fiber; Ti:S, Ti:sapphire. 
fiber, as indicated in Fig. 1. Figure 2 schematically displays the optical domain near $1064 \mathrm{~nm}$ when the $\mathrm{I}_{2^{-}}$ stabilized cw Nd:YAG laser has a set of XPM-induced sidebands spaced by $\Omega_{r}$ and the second, probe, Nd:YAG laser is detuned by $\delta$ from first Nd:YAG laser. From Fig. 2 it is clear that the probe laser creates additional tones in the rf spectrum, offset by $\delta$, on both sides of the $\Omega_{r}$ harmonics. In the observed $\mathrm{rf}$ spectrum, shown in Fig. $3, \Omega_{r} \approx 90 \mathrm{MHz}$ and $\delta=12 \mathrm{MHz}$. The inset of Fig. 3 shows the direct beat between the Nd:YAG lasers and confirms that they are detuned by $12 \mathrm{MHz}$ from each other. The modes labeled $5^{\text {th }}$ and $6^{\text {th }}$ are the fifth and sixth harmonics of the $90-\mathrm{MHz}$ frequency comb at $1064 \mathrm{~nm}$. The $12-\mathrm{MHz}$ spacing of the additional beats from the $90-\mathrm{MHz}$ harmonics verifies that the optical domain presented in Fig. 2 is correct and that XPM is responsible for creating the frequency comb at $1064 \mathrm{~nm}$. If FWM were responsible for generating the frequency comb, the spacing of the heterodyne beats shown in Fig. 3 would not necessarily be $12 \mathrm{MHz}$ and, furthermore, spacing would also depend on the absolute frequency position of the fs comb. No such dependence of the heterodyne beat is observed, further confirming that the frequency comb is created by XPM. This result is not unexpected because of the required phase-matching conditions of FWM.

We can measure the power of the frequency comb as a function of frequency by detuning the probe laser from the comb center and measuring the amplitude of the heterodyne beat (assuming constant probe laser power); these results are given in Fig. 4. A maximum detuning of $15 \mathrm{GHz}$ is obtained before the probe laser mode hops. Although the comb power decreases by $15 \mathrm{~dB}$ at a maximum detuning of $15 \mathrm{GHz}$, there is still a 20-dB signal-to-noise ratio at this point.

Usually the electro-optic crystal in OFC's is driven with a sinusoid, causing the frequency sidebands to have a Bessel function dependence. The resultant comb width can be calculated analytically. In the case of XPM-generated frequency combs, the phasemodulation function is a series of narrow pulses with an initial duty cycle of $10^{-6}$ (10 fs/10 ns) (Ref. 9) for which we are unaware of an analytical solution for the sideband formation. Instead, we perform a numerical calculation. ${ }^{5}$ With the pulse train represented by a series of delta functions, the phase modulation is

$$
\phi_{\mathrm{mod}}=\frac{2 \omega n_{2} z}{c} I \sum_{n=0}^{\infty} \delta(t-n T),
$$

where $\omega$ is the cw carrier frequency, $z$ is the interaction length, $n_{2}$ is the nonlinear index, $I$ is the peak intensity, and $T$ is the period of the pulse train. In reality the pulses have finite width and height. We include these characteristics in the model by expanding the pulse train as a Fourier series and then truncating the series at $N$ terms. For large $N$, the pulse width relative to its period goes as $1 / N$. Consequently, truncating the Fourier expansion at $N=10^{6}$ gives a duty cycle of $10^{-6}$, which matches the initial experimental conditions. Each of these individual Fourier components has its own modulation effects that can be calculated individually. Specifically, each harmonic produces a set of sidebands with a Bessel function amplitude dependence and an adjacent sideband frequency spacing that corresponds to the order of the particular harmonic. This calculation is performed by addition of the effect of each successive harmonic one at a time. We then find the final sideband structure

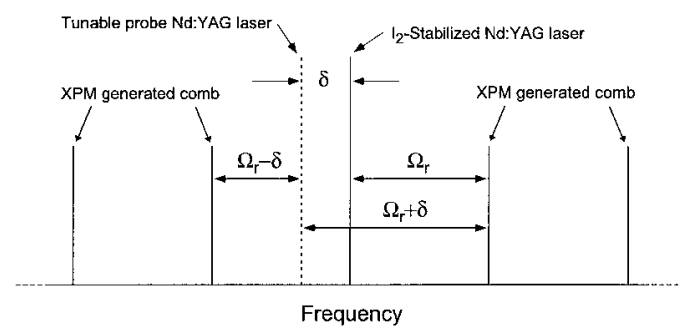

Fig. 2. Optical frequency domain with probe laser and XPM-generated frequency comb. $\Omega_{r}$, pulse-repetition frequency; $\delta$, detuning between two Nd:YAG lasers.

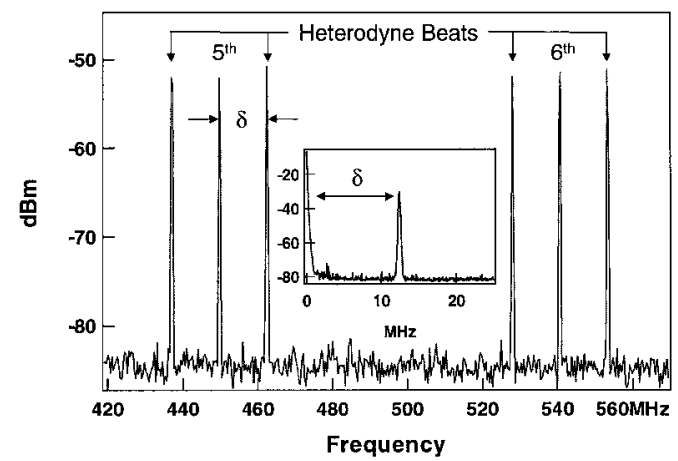

Fig. 3. rf spectrum at $1064 \mathrm{~nm}$. The fifth and sixth harmonics of the $90-\mathrm{MHz}$ frequency comb are shown, along with the heterodyne beats (spaced $12 \mathrm{MHz}$ from the harmonics) between the probe Nd:YAG laser and the frequency comb. Inset, direct heterodyne beat between the two Nd:YAG lasers, showing that they are detuned from each other by $12 \mathrm{MHz}$. The resolution bandwidth is $100 \mathrm{kHz}$.

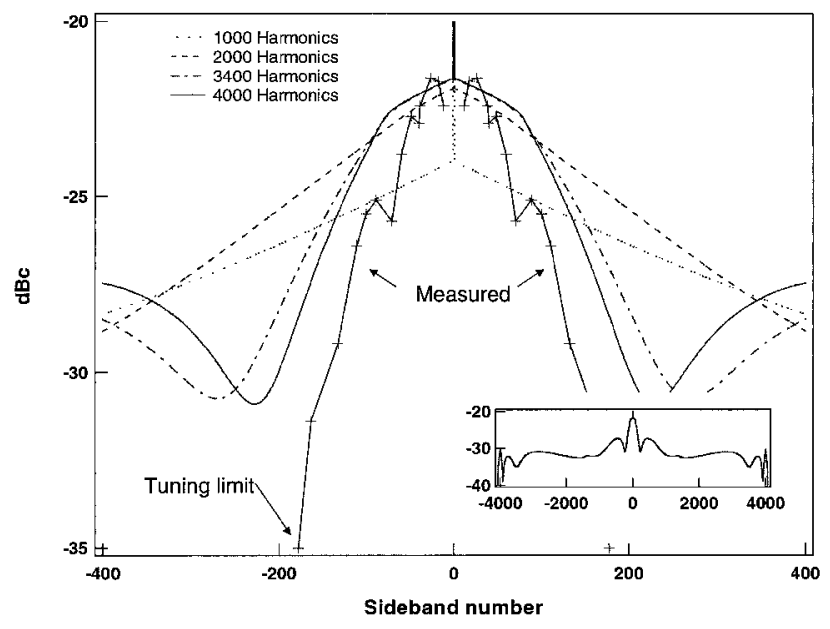

Fig. 4. Results from a numerical simulation of a XPMgenerated frequency comb with femtosecond pulses displaying sideband amplitude envelope versus sideband number. The fs pulse trains are approximated with 1000, 2000, 3000, and 4000 harmonics. Also included is an experimental measurement of the sideband amplitude (shown symmetrically about the carrier). Inset, expanded version of the 4000 harmonic expansion, indicating a relative constant comb amplitude over 7000 sidebands. 
by taking the product of all the sums of the sideband components for each harmonic being considered.

Using experimental parameters, we find the peak modulation depth to be $2 \omega n_{2} z I / c=3,800$, which gives a Fourier amplitude for all the harmonics of $1.9 \times 10^{-3}$. A numerical investigation of the model indicates that this value is crucial in determining the magnitude difference between the carrier and the beginning of the sideband envelope. Interestingly, as harmonics are added to the model this difference soon appears to asymptotically approach a fixed value, as shown in Fig. 4. Experimental results are also included in Fig. 4 and agree extremely well with the numerically determined comb height relative to the carrier.

To obtain a truly realistic model of the fs pulse train requires approximately $10^{6}$ harmonics, and lack of computing resources prevented us from going beyond 4000 harmonics. However, as shown in Fig. 4, with an increasing number of harmonics in the numerical model the sideband envelope converges to the shape of our experimental measurement. Furthermore, with 4000 harmonics, the model predicts a broadband comb envelope spanning 3500 sidebands, or $630 \mathrm{GHz}$ with the present laser. Ultimately, the comb width will be limited by one of three effects: phase-velocity mismatch between the carrier frequency and the $n$th sideband of the comb, group-velocity mismatch between the fs pulse and the pulse generated by the comb, and group-velocity dispersion of the individual fs modes. These characteristics were not included in the numerical modeling. For the present fiber, we believe that the first of these effects limits the comb width to roughly 2000 sidebands.

Clearly, realization of a significantly larger comb width will accelerate the interest and application of XPM-generated frequency combs. If one could scale the pulse repetition rate to the fs laser up to $1-5 \mathrm{GHz}$ (Ref. 10) while maintaining the effective modulation index, a modest width of 1000 sideband lines could span nearly $5 \mathrm{THz}$, a width that approaches the performance of conventional OFC's.

To be useful in frequency metrology, the XPMinduced comb must have a uniform spacing equal to the pulse repetition frequency of the fs laser. The spectral comb uniformity of a 70-fs pulse train emitted directly from an oscillator has been reported to be constant within 3 parts in $10^{17} .{ }^{7}$ However, the possible introduction of timing jitter by the optical fiber is an obvious concern. The comb spacing measured at two spectral extremes of a pulse train broadened in optical fiber by self-phase modulation was shown to be constant to at least a level of $1.9 \times 10^{-12}, 4$ and a comparable level of performance for the XPM-generated frequency comb is expected. Using the quadrature mixing technique reported in Ref. 4, we calculate a worst case error of $2.2 \times 10^{-12}$ between the generated comb spacing and the fs pulse frequency. A more typical slope of the phase drift improves this upper bound by a factor of 3 . No attempt was made to thermally stabilize the input leads to the balanced mixer, so this is an extremely generous estimate of the upper bound.
To demonstrate a possible application of this frequency comb, we measured the detuning between the probe Nd:YAG laser and the $\mathrm{I}_{2}$-stabilized $\mathrm{cw} \mathrm{Nd}$ :YAG and compared it with a second measurement by using a fast photodiode. If $f_{d}$ is the detuning between the two lasers, then $f_{d}=n \Omega_{r} \pm \Delta f$, where $n$ is the number of comb modes and $\Delta f$ is the heterodyne beat. The sign ambiguity of $\Delta f$ is due to the fact that it is not known a priori whether the individual frequency comb member closest to the probe laser is at a higher or lower frequency. Measurement of a 6-GHz detuning by both methods revealed a discrepancy within the frequency drift of the unstabilized probe Nd:YAG laser.

The flexibility offered by this comb-generation technique is shown in a second experiment in which a frequency comb was placed on a single-frequency, frequency-doubled $\mathrm{Nd}: \mathrm{YVO}_{4}$ laser operating at $523 \mathrm{~nm}$. In a manner nearly indentical to the procedure described above (and depicted in Fig. 1), approximately $1 \mathrm{~mW}$ of 532-nm light is copropagated with fs pulses through an optical fiber. After the fiber output was spectrally resolved, a $90-\mathrm{MHz}$ frequency comb centered at $532 \mathrm{~nm}$ was observed.

In summary, we have demonstrated a new application of ultrafast science to optical frequency metrology. Optical frequency combs created by fs pulses and XPM in optical fiber offer the flexibility of nearly arbitrary center frequencies. The possibility of expanding the comb width toward the terahertz level could bridge frequency gaps that are not currently accessible by conventional OFC's.

Funding for this study was provided by the National Institute of Standards and Technology and the National Science Foundation. D. J. Jones and S. A. Diddams are grateful for the support of the National Research Council. D. J. Jones's e-mail address is djjones@jila.colorado.edu.

\section{References}

1. M. Kourogi, K. Nakagawa, and M. Ohtsu, IEEE J. Quantum Electron. 29, 2693 (1993).

2. L. R. Brothers, D. Lee, and N. C. Wong, Opt. Lett. 19, 245 (1994)

3. Th. Udem, J. Reichert, R. Holzwarth, and T. W. Hänsch, Phys. Rev. Lett. 82, 3568 (1999).

4. S. A. Diddams, D. J. Jones, L.-S. Ma, S. T. Cundiff, and J. L. Hall, Opt. Lett. 25, 186 (2000).

5. G. P. Agrawal, Nonlinear Fiber Optics (Academic, San Diego, Calif., 1995).

6. M. T. Asaki, C.-P. Huang, D. Garvey, J. Zhou, H. C. Kapteyn, and M. M. Murnane, Opt. Lett. 18, 977 (1993).

7. Th. Udem, J. Reichert, R. Holzwarth, and T. W. Hänsch, Opt. Lett. 24, 881 (1999).

8. J. L. Hall, L.-S. Ma, M. Taubman, B. Tiemann, F.-L. Hong, O. Pfister, and J. Ye, IEEE Trans. Instrum. Meas. 48, 583 (1999).

9. Because of dispersion in the fiber, the pulse will broaden as it propagates, thus changing the duty cycle.

10. A. Bartels, T. Dekorsy, and H. Kurz, Opt. Lett. 24, 996 (1999). 